\title{
Steigerung der Ausdauerleistungsfähigkeit von durchschnittlich trainierten Personen durch natürliche Sonnenstrahlung (Heliotherapie)
}

\author{
A. Schuh, W. Kneist ${ }^{*}, H$. J. Schmitt \\ Institut für Medizinische Balneologie und Klimatologie der Universität München (Vorstand: Prof. Dr. med. E. Senn) \\ "Klinik für Dermatologie und Allergie Davos (Direktor: Prof. Dr. med. Dr. phil. S. Borelli)
}

\begin{abstract}
Natural sunlight (heliotherapy) causes an increase of endurance capacity in average trained persons
\end{abstract}

Objective: Artificial UV radiation has a positive effect on endurance capacity; several experimental studies have proved this. Based on these results, the conclusion is discussed that UV radiation induces the same metabolic effects as endurance training. Additionally there is no knowledge about the use of natural sunlight and training effects.

Subject: The goal of this study was therefore to quantify the improvement of aerobic performance by heliotherapy. Especially the metabolic effects were investigated.

Design: The study was conducted during a natural heliotherapy lasting 3 weeks, in Davos. 53 patients with atopic neurodermatitis participated. The patients were randomized and put into therapy groups: Under otherwise similar therapeutical conditions, the patients of the "sun-group" ( $n=$ 32) spent an average of 19.3 hours in the sun, the patients of the control group $(\mathrm{n}=21)$ only 5.7 hours. At the beginning and at the end of the therapy, the increase of endurance capacity was measured using bicycle ergometry, measuring parameters among others were pulse rate and lactate level. Other activities like sports on their own were taken down in a diary.

Results: Concerning the lactate values, the results show a significant increase of endurance capacity in the "sun-group" in comparison with the control group $(0.5$ $\mathrm{mmol} / \mathrm{l}, \mathrm{p}<0.01)$ at the same load. The difference of the pulse rate was $3 / \min$ (n.s.)

Conclusion: A slight increase of endurance capacity, especially an adaptation of muscle metabolism, after a three-week heliotherapy was proven. This increase of capacity can be reached not only by artificial radiation but also by natural heliotherapy.

\section{Key words}

Heliotherapy - natural UV-radiation - endurance capacity - muscle metabolism

\section{Einleitung}

Der UV-Strahlung werden zahlreiche positive Auswirkungen z.B. auf Vitamin-D-Synthese, Knochenstoffwechsel, Immunsystem sowie auf verschiedene Kreislaufparameter zugeschrieben (Übersicht in Bühring et al. 1992). Vor allem in der Dermatologie werden neben der Anwendung der künstlichen Strahlung die Patienten mit Psoriasis, Akne und Neurodermitis dem natürlichen Sonnenlicht exponiert. In letzter Zeit wird auch in der Sportmedizin über eine Beeinflussung der Leistungsfähigkeit durch künstliche UV-Bestrahlung diskutiert (Rummel et al. 1991).

Zahlreiche experimentelle Studien mit künstlicher Bestrahlung bestätigen eine Steigerung der Ausdauerleistungsfähigkeit: Bühring (1986) fand eine Abnahme der Herzfrequenz in Ruhe und bei Belastung nach serieller UVB-Bestrahlung. Zusätzlich konnten Roediger et al. (1984) eine gesteigerte Sauerstoffaufnahme nachweisen. Humpeler et al.
(1982) fanden eine vermehrte Sauerstoffabgabe der Erythrozyten an die Mitochondrien. Andere Arbeitsgruppen haben auch einen im Blut angestiegenen Sauerstoffpartialdruck, eine Abnahme des Laktats bei Belastung und eine Verbesserung der Fließeigenschaften des Blutes gefunden (Bäumler et al. 1985, Bühring 1986, Scherf 1986). Aufgrund all dieser Ergebnisse kann vermutet werden, daß UV-Bestrahlung die gleichen Adaptationen im Energiestoffwechsel induziert wie ein Ausdauertraining.

Diese Ergebnisse wurden allerdings nur mit Strahlungsspektren erzielt, die einen großen UVB-Anteil enthielten; mit reiner UVA-, Infrarot- oder sichtbarer Strahlung konnte keine Steigerung der Ausdauerleistungsfähigkeit festgestellt werden (Meffert et al. 1990). Da jedoch zahlreiche der übrigen positiven physiologischen bzw. therapeutischen Effekte wie z.B. Hautzustandsverbesserungen auch nach natürlicher Sonnenstrahlung gefunden werden konnten (Borelli et al. 1981), bleibt nun noch zu klären, ob die leistungssteigernden Effekte nicht doch auch durch natürliches Sonnenlicht, welches wesentlich weniger UVB enthält, hervorgerufen werden können. Es ist somit das Ziel der vorliegenden Studie, die Auswirkungen der natürlichen Sonnenbestrahlung während Heliothe- 


\section{Kurzfassung}

Problemstellung: Der UV-Strahlung wird unter anderem eine Steigerung der Ausdauerleistungsfähigkeit zugeschrieben; zahlreiche experimentelle Studien mit künstlicher Bestrahlung bestätigen dies. Aufgnund dieser Ergebnisse wird diskutiert, daß UV-Bestrahlung die gleichen Stoffwechselprozesse induziert wie ein Ausdauertraining. Diese Resultate wurden jedoch nur mit UVB-Bestrahlung erzielt; mit UVA-, Infrarot- oder sichtbarer Strahlung konnte keine Steigerung der Leistungsfähigkeit festgestellt werden. Es ist deshalb zusätzlich zu klären, ob die leistungssteigernden Effekte auch durch natürliches Sonnenlicht, welches wesentlich weniger UVB enthält, hervorgerufen werden können.

Gegenstand: Ziel der vorliegenden Studie war, die Auswirkungen der natürlichen Sonnenbestrahlung während Heliotherapie auf das Training der Ausdauerleistungsfähigkeit zu untersuchen. Insbesonders standen die metabolischen Effekte im Vordergrund der Untersuchung.

Versuchsplan: In Davos wurden 53 Neurodermitispatienten, die vom Herz-Kreislauf-System her gesund und durchschnittlich trainiert waren, einer 3wöchigen Heliotherapie unterzogen. Bei im übrigen gleichem Therapieregime wurden die Patienten zwei unterschiedlichen Therapiegruppen zugeteilt: Die 32 Patienten der ,Sonnengruppe" haben während der 21 tägigen Studiendauer im Mittel 19,3 Stunden, die
Patienten der Kontrollgruppe dagegen nur 5,7 Stunden unbekleidet in der Sonne gelegen. Die Besonnung fand unter kontrollierten Bedingungen statt. Am Anfang und Ende der Kur wurde die Leistungssteigerung mittels Fahrradergometrie bestimmt. Meßparameter waren in erster Linie Puls und Laktat. Zusätzliche Aktivitäten der Patienten wurden in einem Tagebuch protokolliert.

Ergebnisse: Bei der Sonnengruppe ergab sich anhand der Laktatwerte eine signifikante Verbesserung des aeroben Metabolismus bei gleicher, submaximaler Belastung im Vergleich zur Kontrollgruppe (Differenz zwischen den Gruppen 0,5 mmol / $, \mathrm{p}<0,01$ ). Die Pulswerte beider Gruppen unterscheiden sich am Kurende nur geringfügig ( $3 / \mathrm{min}, \mathrm{n}$. s.).

Schlußfolgerung: Diese Ergebnisse bestätigen, daß durch UV-Bestrahlung dieselben Adaptationen im Muskelstoffwechsel wie durch ein mäßig dosiertes Ausdauertraining hervorgerufen werden. Auch die Größenordnung ist vergleichbar. Diese leichte Steigerung der Ausdauerleistungsfähigkeit läßt sich darüber hinaus nicht nur durch serielle künstliche UVB-Bestrahlung, sondern auch durch eine 3 wöchige Heliotherapie erreichen.

\section{Schlüsselwörter}

Heliotherapie -- natürliche UV-Strahlung Ausdauerleistungs fähigkeit - Muskelstoffwechsel rapie auf das metabolische und physiologische Verhalten unter Ausdauerbelastung zu untersuchen.

\section{Methodik}

An der Studie zur Überprüfung der Auswirkung der Sonnenbestrahlung auf den aeroben Metabolismus haben insgesamt 53 Patienten der Klinik für Dermatologie und Allergie in Davos teilgenommen. Es handelte sich um 20 Männer und 30 Frauen im Durchschnittsalter von 26,7 Jahren. Alle Patienten litten an einer Neurodermitis, waren herzkreislaufgesund, normalgewichtig (mittlere Größe $172 \mathrm{~cm}$, Gewicht 70,0 $\mathrm{kg}$ ) und durchschnittlich trainiert (Laktat $4,7 \mathrm{mmol} / \mathrm{l}$ bei Fahrradergometrie mit im Mittel 125 Watt; Übereinstimmung mit Referenzwerten nach Keul et al. 1970). Ausschlußkriterium stellt eine Sonnenallergie bzw. eine erhöhte Sonnenempfindlichkeit dar.

Die Probanden wurden nach Austestung der minimalen Erythemdosis mittels einer Saalmann-Lichttreppe randomisiert in zwei Gruppen aufgeteilt und einer 3wöchigen Heliotherapie unterzogen; 32 Patienten lagen zwischen 10 und 39 Stunden unbekleidet in der Sonne und bildeten die Sonnengruppe. Unter sonst gleichem therapeutischen Regime bildeten die übrigen 21 Patienten die Kontrollgruppe; sie durften während den 3 Wochen nicht mehr als insgesamt 10 Stunden unbekleidet sonnen. Im Durchschnitt exponierten sich die Patienten der Sonnengruppe während der 21 tägigen Therapic 19,3 Stunden in der Sonne, die Patienten der Kontrollgruppe dagegen nur 5,7 Stunden. Die Sonnenexposition fand während der Monate März/April sowic August/September 1991, immer zur Mittagszeit, auf dem Dachgarten der Klinik in Davos statt.
In einem Tagebuch wurden sämtliche zusätzlichen Tätigkeiten wie Aufenthalte im Freien und Spaziergänge protokolliert; die sportlichen Aktivitäten fanden im Rahmen des Therapieprogramms der Klinik statt und wurden in beiden Gruppen gleich gehalten. Den Patienten wurde vor Beginn der Studie die Empfehlung gegeben, sich zusätzlich nicht der Sonne zu exponieren. Am Anfang der Therapie sowie nach $21 \mathrm{Ta}-$ gen wurde die Ausdauerleistungsfähigkeit während einer Fahrradergometrie (25-Watt-Stufen, je 3 Minuten, Steigerung bis 200 Watt bzw. subjektiver Erschöpfung) erfaßt. Meßparameter waren Puls und Milchsäurespiegel in Ruhe und bei der individuell höchsten Belastungsstufe. Die Belastungsstufen blieben bei den einzelnen Patienten am Ende dieselben wie am Anfang. Die statistische Auswertung wurde mit dem Student-t-Test für paarige Verbindungen vorgenommen. Der Irrtumswahrscheinlichkeit wurde das Signifikanzniveau von $\leq 1 \%\left(\mathrm{p}<0,01^{*}\right) \mathrm{zu}-$ grunde gelegt.

\section{Ergebnisse}

Die durchschnittliche Herzfrequenz (Abb.1) der Sonnengruppe steigt unter Belastung am Therapieanfang vom Ruhezustand mit $85 / \mathrm{min}$ auf $165 / \mathrm{min}$ bei der individuell höchsten Stufe an. Nach 3 Wochen erreicht die Herzfrequenz auf derselben Höchstbelastungsstufe $160 / \mathrm{min}$, d.h., sie steigt um $5 /$ min signifikant $(p<0,01)$ weniger stark an. Die Ruhewerte liegen am Kurende bei $83 / \mathrm{min}$ (n.s.). In der Kontrollgruppe erreicht die durchschnittliche Herzfrequenz, ausgehend von $84 / \mathrm{min}$ am Kuranfang, ebenfalls $165 / \mathrm{min}$; am Therapieende steigt der Puls bei gleicher Belastung um 2/min weniger an, d.h., auf 163/min (n.s.). Der Unterschied der Pulswerte zwi- 
schen beiden Gruppen von 3/min am Kurende kann statistisch nicht gesichert werden.

Der Milchsäurespiegel (Abb.2) steigt bei der Sonnengruppe am Kuranfang, ausgehend von einem Ruhewert von $1,5 \mathrm{mmol} / \mathrm{l}$, auf $4,7 \mathrm{mmol} / \mathrm{l}$ unter der individuell höchsten Belastung an. Am Kurende beträgt der Milchsäurespiegel bei derselben Stufe nur noch 4,2 mmol/1. Der Unterschied von 0,5 $\mathrm{mmol} / \mathrm{l}$ zwischen Therapieanfang und -ende ist signifikant $(\mathrm{p}<0,01)$.

Bei der Kontrollgruppe liegt der Laktatspiegel der höchsten Belastungsstufe bei $4,6 \mathrm{mmol} / \mathrm{l}$; am Therapieende steigt er bei gleicher Belastung auf $4,7 \mathrm{mmol} / \mathrm{l}$. Die Ruhewerte bleiben gleich. Der Unterschied der Belastungswerte am Therapieende zwischen beiden Gruppen ist signifikant $(0,5 \mathrm{mmol} / \mathrm{l}$, $\mathrm{p}<0,01$ ).

\section{Diskussion \\ Muskelmetabolismus}

Die Ergebnisse zeigen aufgrund des Verhaltens des Milchsäurespiegels unter Fahrradergometerbelastung bei der Sonnengruppe im Vergleich zur Kontrollgruppe eine signifikante Zunahme der Ausdauerleistungsfähigkeit.

Dieses Ergebnis steht im Einklang mit den Resultaten der meisten einschlägigen Studien (Überblick in Bühring et al. 1992), unterscheidet sich jedoch von demjenigen der Arbeitsgruppe um Rummel (1991): Die Autoren fanden - gemessen an der aerob/anaeroben Schwelle von $4 \mathrm{mmol} / \mathrm{l}$ - keine Leistungssteigerung aufgrund serieller UVB-Bestrahlung. Sie führten allerdings ihre Untersuchung bei starker körperlicher Belastung durch und werteten die geleisteten Wattzahlen sowohl bei einer Herzfrequenz von 170/min als auch bei einem Laktatspiegel von $4 \mathrm{mmol} / \mathrm{l}$ aus. Da die Ergebnisse der anderen Arbeitsgruppen bei mäßiger körperlicher Belastung gewonnen wurden, kommen die Autoren zu dem Schluß, daß die UVB-Exposition mehr die „vegetative Regulation“ als die metabolische Leistungsfähigkeit zu verbessern scheint. Die Laktatveränderung der vorliegenden Studie, bei der während der Ergometrie ebenfalls hohe Herzfrequenzen $(165 / \mathrm{min})$ erreicht sowie die aerob/anaerobe Schwelle überschritten wurde $(4,7 \mathrm{mmol} / \mathrm{l}$, vgl. Abb. 1 u. 2), spricht jedoch deutlich für eine Beeinflussung des Energiestoffwechsels des Muskels. Diese Schlußfolgerung wird zusätzlich durch das Ergebnis untermauert, daß die durch das vegetative Nervensystem regulierte Herzfrequenz unter Belastung während der Therapie keine nennenswerte Änderung erfährt; auch die von Rummel et al. (1991) beschriebene Ruhebradykardie nach serieller UVB-Bestrahlung konnte nicht gefunden werden. Durch UV-Bestrahlung werden somit Adaptationen im Muskelstoffwechsel hervorgerufen, welche die aerobe Kapazität vergrößern.

\section{Größenordnung der Leistungssteigerung}

Die Verringerung des Laktatspiegels unter individuell höchster Belastung um $0,5 \mathrm{mmol} / 1$ durch die Heliotherapie liegt in derselben Größenordnung wie der Effekt, der durch ein leichtes Ausdauertraining erzielt werden kann: In einer früheren Arbeit (Schuh 1989) wurden bereits die Resultate von 34 Neurodermitispatienten derselben Davoser Klinik, wel-

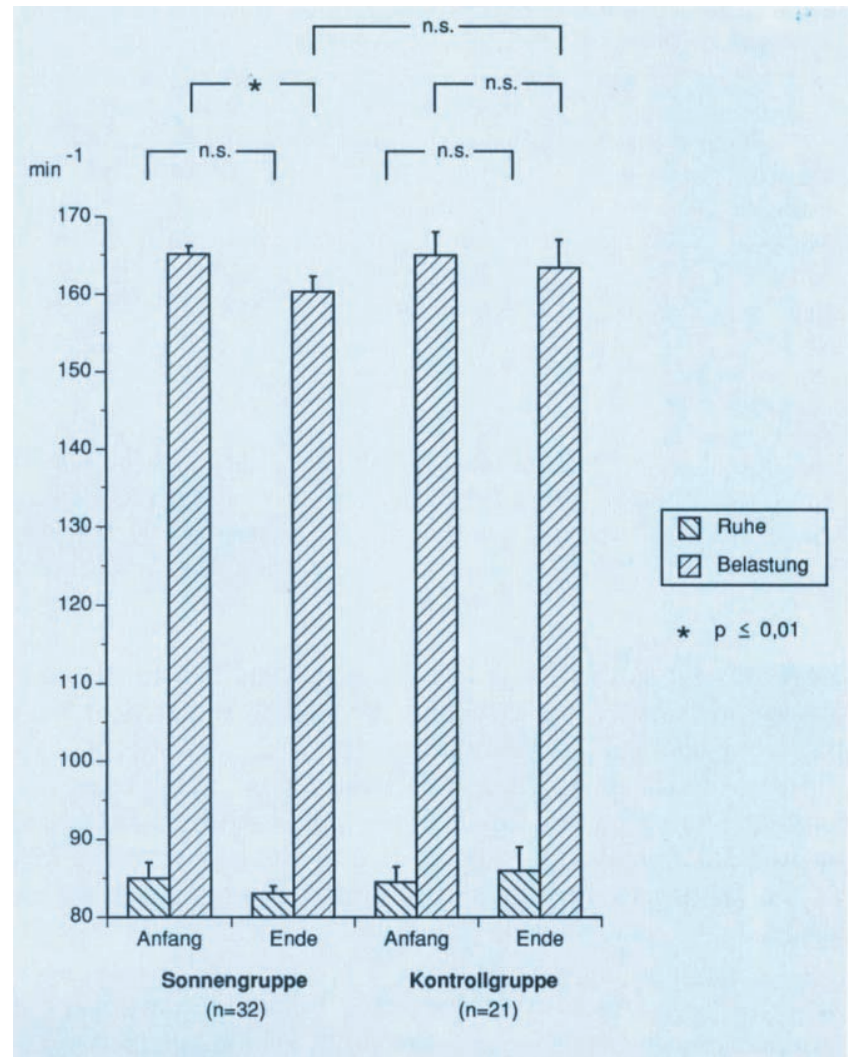

Abb. 1 Herzfrequenz der Sonnengruppe $(n=32)$ und der Kontrollgruppe ( $n=21)$ während Fahrradergometrie in Ruhe und während höchster erreichter Belastung am Therapieanfang und nach 3 Wochen Heliotherapie.

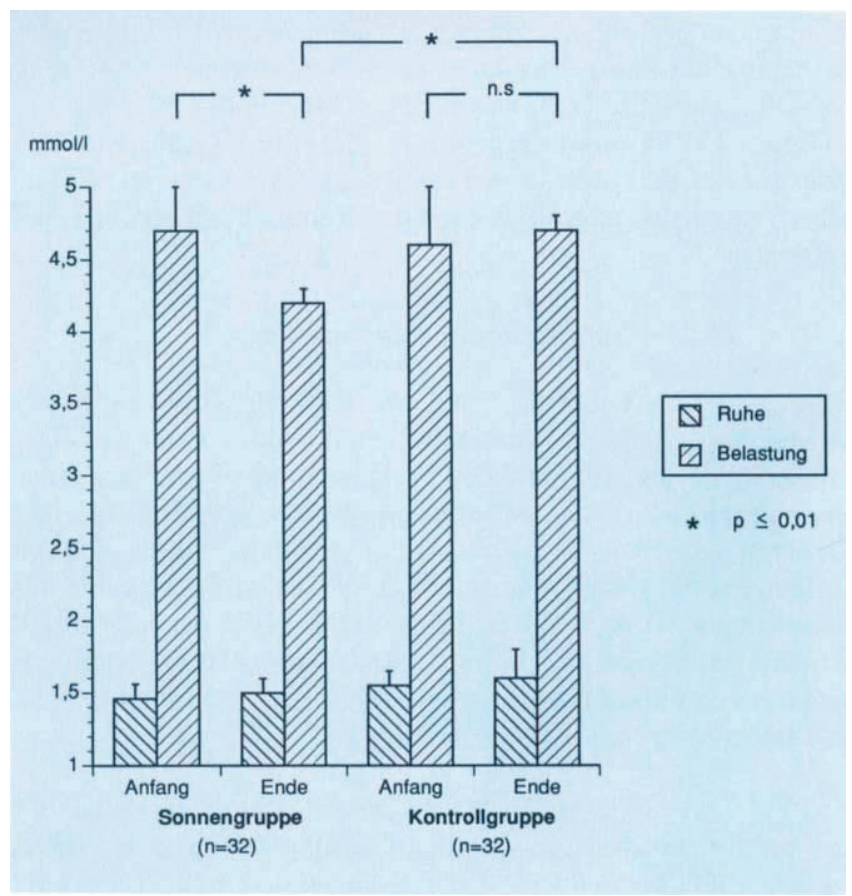

Abb. 2 Milchsäurespiegel der Sonnengruppe $(n=32)$ und der Kontrollgruppe $(n=21)$ während Fahrradergometrie in Ruhe und während höchster erreichter Belastung am Therapieanfang und nach 3 Wochen Heliotherapie. 
Tab. 1 Durchschnittliche natürliche UV-Dosis in Davos von 1987, Summen pro Monat (nach Vocks et al. 1989).

\begin{tabular}{lcc}
\hline & UV-B $\left(\mathrm{J} / \mathrm{cm}^{2}\right)$ & UV-A $\left(\mathrm{J} / \mathrm{cm}^{2}\right)$ \\
\hline Januar & 1,25 & 127,38 \\
Februar & 2,10 & 214,00 \\
März & 4,37 & 200,54 \\
April & 7,81 & 358,02 \\
Mai & 14,43 & 660,96 \\
Juni & 8,39 & 228,11 \\
Juli & 9,45 & 272,00 \\
August & 7,34 & 211,30 \\
September & 9,41 & 198,27 \\
Oktober & 2,94 & 101,98 \\
November & 1,40 & 47,73 \\
Dezember & 1,00 & 101,50 \\
\hline
\end{tabular}

che vom Alter, Gewicht und Trainingszustand her mit den der vorliegenden Studie untersuchten Personen vergleichbar sind und ein 3wöchiges Terraintraining (4mal die Woche, jeweils 20-40 Minuten ansteigend, bei Herzfrequenz 180 minus Lebensalter) durchführten, dokumentiert; die Patienten verbesserten sich um einen vergleichbaren Betrag von $0,45 \mathrm{mmol} / \mathrm{l}$, wobei die Ergebnisse mit demselben Untersuchungsdesign erfaßt wurden.

Auch in der kardialen Rehabilitation führt ein Ausdauertraining größenordnungsmäßig zu ähnlichen Anpassungen: Liesen u. Mitarb. (1981) erreichten nach einem 3monatigen leichten Ausdauertrainingsprogramm mit Herzinfarktpatienten bei Fahrradergometerbelastung eine zwischen 0,4 $\mathrm{mmol} / 1$ (bei 100 Watt) und $1,0 \mathrm{mmol} / \mathrm{l}$ (bei 150 Watt) signifikant niedrigere Laktatbildung. Allerdings zeigte sich hier der Trainingseffekt auch bezüglich der Herzfrequenz: Im Gegensatz zur Heliotherapie konnten die Herzfrequenzen signifikant erniedrigt werden. Die Heliotherapie führt somit zu einer Reduktion des Milchsäurespiegels in derselben Größenordnung wie ein leichtes Ausdauertraining; die Auswirkung auf die Herzfrequenz ist allerdings nach der Heliotherapie deutlich geringer.

\section{Natürliche Sonnenstrahlung}

Die Steigerung der Leistungsfähigkeit wurde in den o.a. Arbeiten immer bei Applikation einer seriellen UVB-Strahlung gefunden; den übrigen Anteilen des Spektrums wird dabei keine biologische Relevanz zugeschrieben. In einer weiteren, von Meffert (1990) durchgeführten Studie wurden Probanden mit Lampen bestrahlt, deren Emission vor allem aus langwelliger UVA-Strahlung bestand. Der UVB-Anteil betrug 8 $\%$. Die Probanden erhielten 4 bzw. 20 Ganzkörperbestrahlungen mit einer kumulativen Dosis von 8,8 bzw. $51,0 \mathrm{~J} / \mathrm{cm}^{2}$, d.h., zwischen 0,7 bzw. $4,1 \mathrm{~J} / \mathrm{cm}^{2}$ UVB.

Die UVB-Dosis beider Bestrahlungsserien, mit der Meffert eine Steigerung der Leistungsfähigkeit erreichte, liegt deutlich höher als diejenige, welche die Patienten der vorliegenden Arbeit während ihrer Heliotherapie in Davos erhielten: Vocks et al. (1989) gelang es in einer Untersuchung zum ersten Mal, die von einzelnen Patienten therapeutisch genutzte Sonnenstrahlung täglich quantitativ zu bestimmen. Danach erhielt ein Patient in Davos im Jahr 1987 in einem Behandlungszeitraum von 6 Wochen bei einer 2stündigen Ganzkörperson- nenexposition täglich, eine therapeutische Gesamtdosis von $1,042 \mathrm{~J} / \mathrm{cm}^{2}$ an natürlicher UVB-Strahlung (berechnet für den Jahresdurchschnitt). Aufgeschlüsselt nach den einzelnen Monaten (Tab. 1) und auf die vorliegende Untersuchung übertragen, hätten die Patienten der Sonnengruppe 1987 während den drei Wochen Heliotherapie bei einer durchschnittlichen Sonnenzeit von 19,3 Stunden eine kumulative UVB-Dosis von $0,4 \mathrm{~J} / \mathrm{cm}^{2}$ erhalten. Dies ist im Vergleich zu der geringsten Bestrahlungsdosis von Meffert $\left(0,7 \mathrm{~J} / \mathrm{cm}^{2}\right)$ deutlich niedriger.

Damit ist verdeutlicht, daß die leistungssteigernden Effekte - hier speziell eine Verbesserung des aeroben Muskelstoffwechsels - auch bei einer wesentlich geringeren UVB-Dosis erzeugt werden können; die Bestrahlung durch das natürliche Sonnenlicht reicht dazu aus.

\section{Höhenabhängigkeit}

Bei der Heliotherapie mit natürlicher Bestrahlung ist jedoch $\mathrm{zu}$ beachten, daß die dargelegten Effekte in Davos $(1785 \mathrm{~m})$ gewonnen wurden, das eine deutlich höhere UV-Intensität aufweist als niedriger gelegene Orte. Damit bleibt die Frage nach der Existenz einer unteren Grenze der Strahlendosis, um den Wirkungsbereich für Heliotherapie zur Steigerung der Ausdauerleistungsfähigkeit noch genauer festzulegen.

Der Einfluß des verringerten Sauerstoffpartialdrucks in der Höhe wirkt verstärkend auf Trainingseffekte (Mairbäurl et al. 1986): Es kommt zu einer Intensivierung der kardiozirkulatorischen Adaptationen und zu signifikanten Leistungsverbesserungen, die sich in einer Vergrößerung der maximalen Sauerstoffaufnahmekapazität sowie in einer Verminderung der Herzfrequenz und des Laktatspiegels bei gegebener Belastung manifestieren. Dieser Hypoxieeffekt läßt sich bei den Patienten der Studie nicht feststellen: Die Kontrollgruppe hat sich in ihrem aeroben Metabolismus nicht verbessert. Da beide Gruppen jedoch dieselbe Aufenthaltsdauer in Davos und eine vergleichbare körperliche Aktivität aufwiesen, muß der Trainingseffekt der Sonnengruppe aufgrund der Heliotherapie und nicht aufgrund einer Höhenakklimatisation zustande gekommen sein.

\section{Schlußfolgerung}

Die Ergebnisse bestätigen somit, daß durch UV-Bestrahlung dieselben Adaptationen im Muskelstoffwechsel wie durch ein leichtes Ausdauertraining hervorgerufen werden. Diese Verbesserung der aeroben Kapazität läßt sich nicht nur mittels serieller künstlicher UVB-Bestrahlung, sondern auch durch eine 3 wöchige Heliotherapie mit natürlicher Sonnenstrahlung erzielen. 


\section{Literatur}

Büumler, H., H. P. Scherf, H. Meffert, D. Lerche, M. Thümmler: Einfluß der Ultraviolett-Ganzkörperbestrahlung auf Fließeigenschaften des Blutes. Dermatol. Monatsschr. 171 (1985) 366-371

Borelli, S., H. Düngemann: Fortschritte der Allergie und Dermatologie I.M.P. Verlagsgesellschaft mbH, Neu-Isenburg 1981

Bühring, M.: Kreislauf- und metabolische Effekte serieller UV-Expositionen. Z. phys. Med. Baln. Med. Klim. 15 (1986) 170-172

Bühring, M., E. G. Jung (Hrsg.): UV-Biologie und Heliotherapie. Hippokrates, Stuttgart 1992

Humpeler, E., P. Mairbäurl, W. Schulz-Amling: Effect of whole body-irradiation on oxygen delivery from the erythrocyte. Eur. J. appl. Physiol. 49 (1982) 209-214

Keul, J., E. Doll, G. Haralambie: Freie Fettsäuren, Glyzerin und Triglyzeride im arteriellen und femoralvenösen Blut vor und nach einem 4wöchigen körperlichen Training. Pflügers Arch. 316 (1970) 194

Liesen, H., W. Hollmann: Ausdauersport und Stoffwechsel. Hoffmann Verlag, Schorndorf 1981

Mairbäurl, H., W. Schobersberger, E. Humpeler, W. Hasibeder, W. Fischer, E. Raas: Beneficial effects of exercising at moderate altitude on red cell oxygen transport and of exercise performance. Pflügers Arch. 406 (1986) 594-599

Meffert, H., H.-P. Scherf, H. Bäumler, N. Sönnichsen: Phototherapie geht auch unter die Haut. Systemische Effekte der Ultraviolett-, sichtbaren bzw. Infrarotstrahlung bei seriellen Ganzkörperbestrahlungen. Dt. Derm. 38 (1990) 1383-1386

Roediger, E., M. Bühring, F. Wolff: Kreislauf-und Stoffwechselparameter bei seriellen Bestrahlungen mit UVA und UVB. Z. phys. Med. Baln. med. Klim. 13 (1984) 34-35

Rummel, M., A. Falkenbach, R. Föhrenbach, D. Böhmer, M. Bühring: Die körperliche Leistungsfähigkeit nach einer seriellen Bestrahlung mit UVB. In: Bernett, P., D. Jeschke: Sport und Medizin Pro und Contra. Zuckschwerdt Verlag, München 1991

Scherf, $H$. .. : Vergleichende Untersuchungen zur Wirkung von Ultraviolettbestrahlung der Haut bzw. des Blutes bei Patienten mit arterieller Verschlußkrankheit, Psoriasis und Gesunden. Med. Promotion, Berlin 1986

Schuh, A.: Das Krankheitsbild des Trainingsmangels und seine Behandlung durch Klimatherapie in Form einer Terrainkur unter kühlen Bedingungen. Habilitationsschrift, Medizinische Fakultät, München 1989

Vocks, E., B. Seifert, H. Hahn, C. Fröhlich: Quantitative Erfassung der Heliotherapie bei Psoriasis vulgaris im Hochgebirgsklima. Zschr. F. Hautkr. 64 (1989) $466-472$
PD Dr. Dr. med. habil. Angela Schuh

Institut für Medizinische Balneologie und Klimatologie der Universität München

Marchioninistraße 17

D-81377 München 